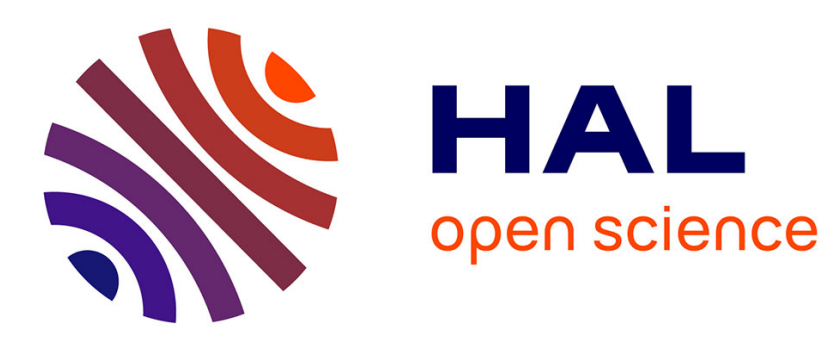

\title{
Le cheminement des étudiants dans les écoles supérieures d'art
}

\author{
Gilles Galodé, Christophe Michaut
}

\section{To cite this version:}

Gilles Galodé, Christophe Michaut. Le cheminement des étudiants dans les écoles supérieures d'art. Revue Française de Pédagogie, 2003. halshs-01225551

\section{HAL Id: halshs-01225551 https://shs.hal.science/halshs-01225551}

Submitted on 6 Nov 2015

HAL is a multi-disciplinary open access archive for the deposit and dissemination of scientific research documents, whether they are published or not. The documents may come from teaching and research institutions in France or abroad, or from public or private research centers.
L'archive ouverte pluridisciplinaire HAL, est destinée au dépôt et à la diffusion de documents scientifiques de niveau recherche, publiés ou non, émanant des établissements d'enseignement et de recherche français ou étrangers, des laboratoires publics ou privés. 


\section{Varia}

\section{Le cheminement des étudiants dans les écoles supérieures d'art

\author{
Gilles Galodé \\ Christophe Michaut
}

L'article porte sur le cheminement des étudiants des écoles supérieures d'art (autrefois écoles des beaux-arts). II s'agit tout d'abord de présenter les singularités de l'enseignement supérieur artistique par rapport aux autres secteurs de l'enseignement supérieur. Dans un deuxième temps, le recensement du recrutement social et scolaire des écoles révèle une forte hétérogénéité des établissements. Enfin, l'analyse longitudinale des parcours de 1570 étudiants inscrits en première année montre que la réussite des études artistiques dépend davantage du contexte d'étude que de l'héritage culturel.

Mots-clés : beaux-arts, réussite scolaire, recrutement, identité artistique, contexte scolaire.

\section{INTRODUCTION}

L'entrée dans une école d'art constitue un choix d'étude original et à certains égards hétérodoxe parmi les formations de l'enseignement supérieur. Plus qu'ailleurs, le choix individuel doit supporter une part d'inconnu et donc d'incertitude. En effet, l'enseignement de l'art fondamentalement paradoxal, ne saurait être normé à l'instar d'autres disciplines. Face à cette difficulté, les écoles supérieures d'art, sous leur appellation ancestrale "Écoles des Beaux-arts ", ont entrepris au cours des deux dernières décennies un processus de transformation radical de leurs cursus. Elles se présentent désormais comme un système de formation homogène et finalisé, répondant en tous points aux critères de l'enseignement supérieur.
Pourtant, à l'heure de l'université de masse, elles constituent encore un segment atypique, mal connu et peu exploré, du système d'enseignement supérieur français.

Ces écoles ne sont pas les seules à proposer un enseignement supérieur artistique. Ce dernier comprend également les universités, les établissements préparant au brevet de technicien supérieur (BTS), au diplôme des métiers d'arts (DMA) ou au diplôme supérieur des arts appliqués (DSAA), et enfin les écoles d'art publique ou privées, dont certaines, délivrant leurs propres diplômes, constituent le lieu d'excellence de leur spécialité. Ces établissements qui assurent des formations à caractère technique relèvent du ministère de l'Éducation Nationale. Ils se distinguent par leur statut, leur cursus, leur recrutement 
et leur degré de spécialisation. Dépendant du ministère de la Culture et de la Communication, les écoles supérieures d'art ont une vocation autre, irréductible aux référents techniques: enseigner l'art. Elles accueillaient, en 1999-2000, 9222 étudiants alors que les autres établissements en accueillaient à la même date près de neuf fois plus (Le Cosquer et Hérault, 2001). Historiquement, les écoles des beauxarts ont émergé de manière spontanée, à partir d'initiative locale, hors de toute carte scolaire ou principe directeur d'accès (Galodé, 1994). Ceci a conduit à un système dual avec deux catégories d'écoles, de statut différent, mais pédagogiquement réunies dans un modèle unique. Les unes, sont des écoles nationales sous tutelle administrative d'État (8 écoles), les autres sont des écoles régionales et municipales de statut territorial (44 écoles), sous tutelle locale. Les écoles territoriales sont financées principalement à l'échelon local, les écoles nationales par l'État. La tutelle pédagogique unique, assurée par l'État, définit les règles de formation applicables à toutes les écoles sans distinction de statut.

L'organisation actuelle des études offre le choix entre un cursus long de cinq ans, largement majoritaire (neuf élèves sur dix) et un cursus professionnel court de trois ans, dans un nombre restreint d'établissements (un quart). La première année propédeutique est commune. Chaque cycle possède un diplôme terminal de fin d'étude (1), et des certifications intermédiaires. Cette organisation des études résulte des réformes du début des années 70 et de la fin des années 80 dont les objectifs visaient d'une part à inscrire pleinement les écoles d'art dans l'enseignement supérieur, d'autre part à élever les niveaux de qualification en vue d'améliorer l'insertion professionnelle des étudiants. Ainsi, les cursus se sont peu à peu homogénéisés et le baccalauréat devient nécessaire (sauf exceptions) pour s'inscrire dans une école. Par ailleurs, la formation artistique délivrée dans ces écoles a profondément évolué en matière de curricula. Jusqu'en 1968, l'apprentissage technique et les exercices de représentation dominent selon le modèle académique. Dans les années 70, l'apprentissage du métier cède le pas et laisse place à une formation centrée sur l'artiste et sur l'expression de sa créativité. Désormais, il s'agit de développer chez les étudiants des attitudes davantage orientées vers la vie culturelle locale et la création internationale, par le biais d'une formation en culture générale et artistique plus prégnante (Liot, 1999).

Enfin, trois écoles phares de statut public occupent, à des titres divers, une situation particulière par rapport à l'ensemble précédent: l'École Nationale Supérieure des Beaux arts de Paris (ENSBA), l'École Nationale Supérieure des Arts Décoratifs (ENSAD) et la toute récente École Nationale Supérieure de Création Industrielle (ENSCI). Tous trois parisiens, ces établissements constituent, chacun avec leurs cursus propres et leur identité historique ou thématique affirmée, le segment élitiste des formations artistiques.

Hormis les études sur les prestigieuses écoles parisiennes et les études de cas sur quelques écoles de province, les recherches sur l'hétérogénéité des écoles sont extrêmement rares. Cet article vise justement à caractériser cette hétérogénéité en privilégiant certaines dimensions de "l'effet établissement » (Cousin, 2000). II ne s'agit pas ici d'étudier la politique des établissements sous l'angle des projets pédagogiques ou des partenariats extérieurs mais d'examiner les politiques de recrutement et de sélection des étudiants. Le cadre plus général du projet (Galodé, 1998) est issu des travaux de la commission nationale de réforme des écoles d'art dont l'objet était une inscription plus effective de cet ensemble parmi les formations de l'enseignement supérieur (Imbert, 1998). Cette recherche de caractère longitudinal a été conduite auprès de 29 écoles (dont l'ENSBA et L'ENSAD), soit la moitié des établissements. Les données portent sur 1570 étudiants inscrits en 1998-1999 en première année d'étude. Interrogés successivement en début d'année scolaire et dans le courant de la première année, les étudiants ont répondu à deux questionnaires portant sur leurs caractéristiques personnelles, leurs conditions de vie, leurs rapports aux études... Par ailleurs, leurs résultats scolaires à l'issue de la première et de la seconde année ont été recueillis. Nous privilégierons ici le cheminement des étudiants.

II s'agira dans un premier temps d'identifier l'hétérogénéité des écoles en examinant leur recrutement social et scolaire. Une seconde partie sera consacrée à la sélectivité des écoles et au cheminement des étudiants. L'analyse visera à déterminer les facteurs associés à la réussite (ou l'échec) de manière à les confronter aux « déterminants » de la réussite universitaire.

\section{LE RECRUTEMENT DES ÉCOLES}

\section{Un recrutement socialement différencié}

Par rapport aux autres secteurs de l'enseignement supérieur, les écoles des beaux-arts ont un recrutement social situé entre celui des classes prépara- 
Tableau I. - Origine socioprofessionnelle des étudiants (en \%)

\begin{tabular}{|l|c|c|c|c|c|}
\hline \multicolumn{1}{|c|}{ Origine socioprofessionnelle } & CPGE & $\begin{array}{c}\text { Écoles } \\
\text { supérieures } \\
\text { d'art }\end{array}$ & $\begin{array}{c}\text { Universités } \\
\text { (1 } 1_{\text {cycle })}\end{array}$ & IUT & STS \\
\hline Professions libérales, cadres supérieurs & 51,4 & 42,7 & 30,4 & 26,5 & 15,1 \\
\hline Professions intermédiaires & 15 & 16,6 & 17,3 & 19,9 & 17,4 \\
\hline Employés & 8,8 & 9,7 & 14,5 & 15,1 & 16,6 \\
\hline Ouvriers & 5,4 & 11,2 & 13,2 & 16,2 & 23,0 \\
\hline Autres PCS & 19,4 & 19,8 & 24,6 & 22,3 & 27,9 \\
\hline Ensemble & 100 & 100 & 100 & 100 & 100 \\
\hline
\end{tabular}

Source : Repères et références statistiques sur les enseignements, la formation et la recherche, édition 2001, p. 179, sauf données sur les écoles supérieures d'art.

toires aux grandes écoles (CPGE) et celui des premiers cycles universitaires. Largement moins populaires que les filières techniques (STS, IUT), les écoles supérieures d'art n'opèrent toutefois pas un recrutement aussi élitiste que les CPGE. Ainsi, $42,7 \%$ des étudiants de première année des beaux-arts ont un père occupant la profession de cadre supérieur tandis que $51,4 \%$ des étudiants des CPGE sont dans cette situation. À un niveau plus fin, on constate que les étudiants dont les parents occupent une profession dans le secteur culturel sont relativement rares $(3,8 \%)$.

Ceci étant, cette répartition globale masque l'extrême hétérogénéité des écoles en matière de recrutement social. La proportion d'étudiants dont le père est cadre supérieur varie de $16,1 \%$ à Fort-deFrance jusqu'à 69,6\% à l'école nationale supérieure des arts décoratifs (ENSAD : Arts-Déco. Paris). Plus précisément, on observe à un pôle les écoles surreprésentées par les enfants de cadres supérieurs (ENSBA, ENSAD, Strasbourg, Angoulême, Montpellier, etc.) et à l'autre pôle les écoles sous-représentées (Dunkerque, Fort-de-France). Évidemment, la représentation sociale des écoles n'est pas indépendante de la géographie sociale des villes et des régions dans lesquelles sont implantées les écoles. Toutefois, certaines villes comme Nantes ou Dijon recrutent relativement peu d'enfants de cadres. À l'inverse, les villes relativement "populaires" comme Angoulême ou Le Havre ont plutôt un recrutement bourgeois. Ceci suggère d'ores et déjà que le recrutement des écoles ne s'effectue pas localement et que leur attractivité repose sur d'autres facteurs que le seul facteur géographique.
Sur l'ensemble des écoles, 46,9\% des étudiants ont obtenu leur baccalauréat dans la même académie que l'école d'art dans laquelle ils sont inscrits. La mobilité des étudiants des classes favorisées est significativement supérieure aux autres étudiants, en particulier les étudiants d'origine populaire. Ainsi, en excluant les étudiants ayant obtenu leur baccalauréat dans la même ville que l'école d'art et lorsque sont contrôlées les différentes écoles, les enfants de cadres supérieurs restent toujours plus mobiles que les autres étudiants (+ 13,9\% par rapport aux enfants d'ouvriers). Ce résultat s'accorde avec l'hypothèse de Boudon selon laquelle les enfants d'origine populaire sont plus sensibles au coût des études et au risque d'échouer, quitte à minorer les bénéfices futurs (Boudon, 1973 ; Duru et Mingat, 1988). Plus encore, la mobilité des étudiants dépend des spécificités des écoles d'arts. Par exemple, seuls $24,4 \%$ des étudiants inscrits à l'école de Tourcoing n'ont pas obtenu leur baccalauréat dans l'académie alors que $86,5 \%$ des étudiants strasbourgeois sont dans cette situation. II y a vraisemblablement un effet de notoriété des écoles qui incite les étudiants à s'inscrire dans des écoles éloignées du domicile parental. Critère qui, selon les étudiants, est l'un des plus importants dans le choix de l'école après celui de la spécialisation offerte.

\section{Les filles plus nombreuses dans les écoles d'art}

En première année, les filles sont plus nombreuses $(60,7 \%)$ que les garçons, sachant néanmoins que tous niveaux confondus, elles sont moins représentées dans les écoles supérieures d'art que dans les 
disciplines universitaires artistiques $(57,6 \%$ vs $65,8 \%$, source : Le Cosquer et Hérault, 2001). Les écoles de Dunkerque, Le Havre et Angoulême attirent davantage de garçons alors qu'à l'opposé Strasbourg, Bourges ou Marseille sont des écoles plutôt "féminines ». Ces différences de composition ne sont pas sans lien avec la nature des formations dispensées: l'art graphique et les technologies audiovisuelles pour les garçons, la décoration et la restauration pour les filles. Ce résultat n'est pas surprenant dans la mesure où de nombreuses recherches montrent que les préférences scolaires sont marquées sexuellement (Duru-Bellat, 1994).

\section{L'importance de la scolarité antérieure sur le recrutement}

Soulignons avant tout que seuls $47,4 \%$ des étudiants s'inscrivent en première année directement après avoir obtenu leur baccalauréat ou son équivalent. 7,3 \% y ont déjà été inscrits, $16,6 \%$ se sont préparés au concours d'entrée, $21,3 \%$ ont suivi auparavant des études et $7,4 \%$ se trouvent dans une autre situation (interruption des études, activité professionnelle, service national, etc.). C'est dire à quel point l'entrée dans une école d'art ne se fait pas automatiquement le diplôme du secondaire acquis. Nombreux sont les étudiants ayant auparavant suivi des études, notamment des études artistiques dans les universités (Histoire des arts et archéologie, Arts plastiques, Arts du spectacle, Musicologie, etc.). Si la préparation aux études artistiques n'est pas formellement indispensable pour entrer dans une école d'art, elle apparaît souvent nécessaire. Près d'un tiers des étudiants a suivi une préparation au concours d'entrée. Ce chiffre masque en réalité une extrême diversité de fréquentation des écoles préparatoires. Ainsi, la proportion d'étudiants y ayant eu recours et qui a réussi le concours d'entrée varie de $4 \%$ à Tourcoing jusqu'à 91,3\% à Art-Déco Paris. Plus généralement, il semble que l'accès aux écoles parisiennes et aux écoles des grandes villes de province nécessite une préparation. À l'inverse, les petites écoles accueillent majoritairement les étudiants n'ayant pas suivi de formation spécifique. Ce sont généralement des étudiants qui n'ont pas, au cours de leurs études secondaires, suivi d'option artistique.

Les chances d'accéder à certaines écoles étant relativement faibles, les étudiants vont multiplier les candidatures. Près des deux tiers se présentent à plusieurs concours (en moyenne trois). II n'existe pas de normes nationales fixant les critères de sélection des étudiants. Certaines écoles vont privilégier le dossier de travaux des candidats, d'autres l'entretien des candidats. Certains enseignants semblent opposés à une sélection sur le dossier scolaire (Sulzer, 1999). Les étudiants estiment d'ailleurs que la sélection s'opère avant tout lors de l'entretien avec les enseignants. Dans certaines écoles, ce ne sont pas les capacités à produire un objet qui sont valorisées mais la production d'un art essentiellement discursif (Liot, 1999). Dès lors, les entretiens revêtent un caractère essentiel au cours desquels devra s'exprimer non pas un « talent artistique " mais bien souvent un « talent social » (Azam, 1998).

Près de la moitié des étudiants détient un baccalauréat littéraire et parmi eux $72 \%$ ont suivi l'une des options artistiques en classe de Terminale (l'équivalent du bac A3). On trouve ensuite, par ordre décroissant, les bacheliers scientifiques (S/C/D/D'/E) $(12,8 \%)$, les bacheliers F11 (musique), F11' (danse) et F12 (arts appliqués) $(8,1 \%)$, les bacheliers ES et B $(7,8 \%)$, les bacheliers professionnels et les détenteurs d'un brevet de technicien (6\%), les autres bacheliers technologiques (STI, STL, SMS, STT) $(5,4 \%)$ et les bacheliers étrangers (4\%). À noter enfin que $8 \%$ des étudiants ne possèdent pas de baccalauréat sachant que celui-ci n'est pas indispensable pour accéder aux écoles artistiques. Sur ce point, il apparaît que cette situation, fréquente au début des années 80 (Galodé, 1994) - environ $40 \%$ ne possédait pas le baccalauréat - est désormais exceptionnelle, le baccalauréat et plus récemment une formation supérieure devenant la norme de recrutement. Là encore, les politiques de recrutement des bacheliers sont extrêmement variées. Certaines écoles recrutent essentiellement des bacheliers littéraires, en particulier ceux qui ont suivi une option artistique (Nice, Fort-de-France). D'autres privilégient les bacheliers technologiques ou professionnels (Limoges, Dunkerque) alors que d'autres recrutent relativement plus de bacheliers ES et $S$ (Reims, Orléans, Arts-Déco. Paris) (2).

Afin de situer les différentes écoles, une analyse factorielle des correspondances multiples a été réalisée dans laquelle figurent les cinq variables suivantes : la profession et la catégorie sociale (PCS) du père, le sexe de l'étudiant, sa série et sa mention du baccalauréat et enfin l'école, introduite comme variable supplémentaire.

Le quadrant inférieur droit regroupe les écoles qui procèdent à un recrutement social et scolaire élitiste (ENSBA, ENSAD, Angoulême, Strasbourg). Les enfants de cadre et les bacheliers $S$ et ES ayant obtenu leur bac avec mention sont effectivement 
Figure 1. - Analyse des correspondances multiples

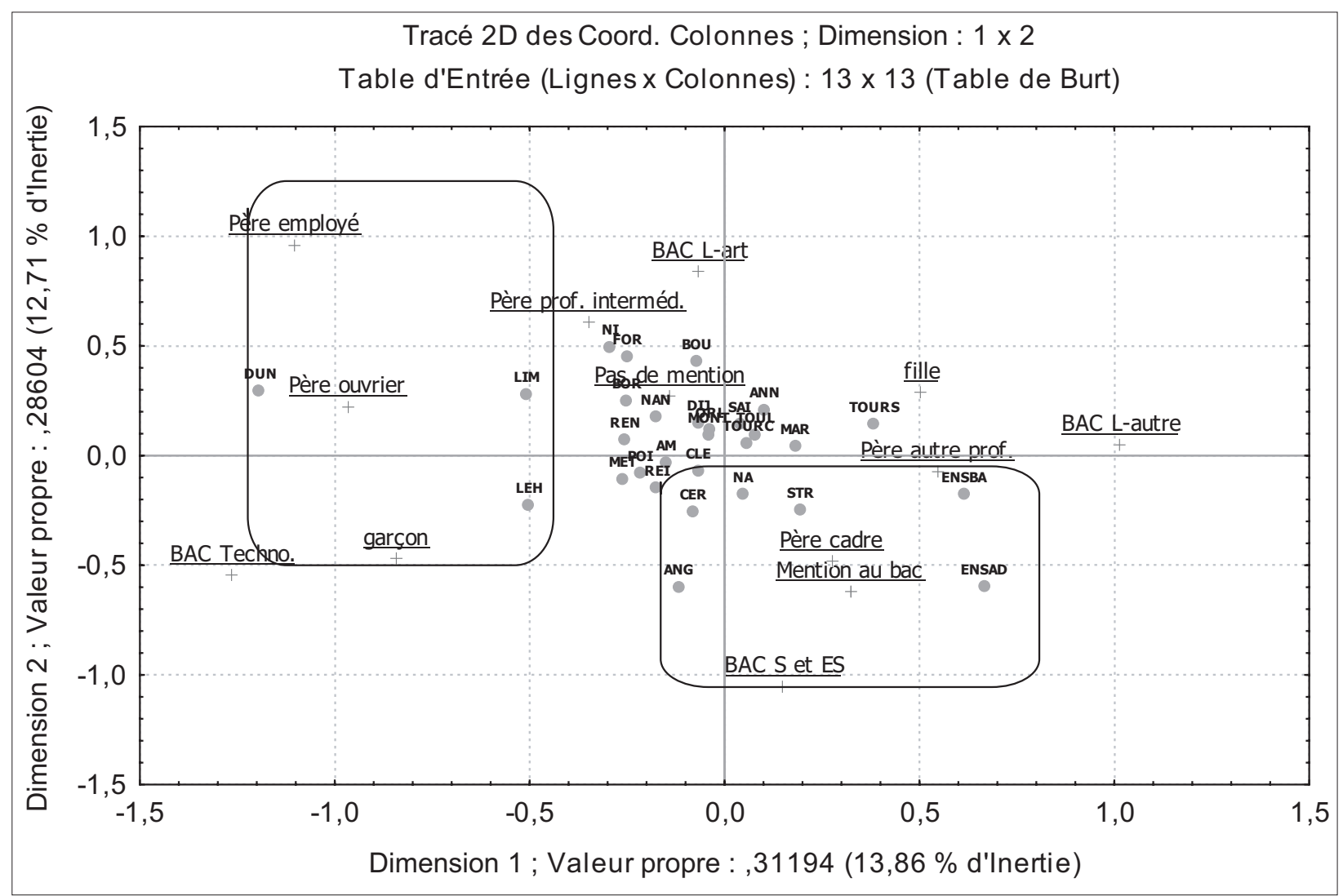

(Souligné : variables actives ; gras : variables supplémentaires)

plus représentés dans ces écoles. À l'inverse, les petites écoles (Dunkerque, Limoge, Le Havre, Fortde-France) recrutent plus souvent des garçons possédant un baccalauréat technologique dont les parents occupent une profession d'employé ou d'ouvrier. En définitive, c'est essentiellement par un processus de sélection à l'entrée et d'auto-sélection que se détermine la hiérarchie sociale des écoles. Une dichotomie apparaît entre les écoles parisiennes qui sur le plan du recrutement social s'apparentent aux grandes écoles (3) et les écoles de province qui procèdent à un recrutement plus populaire.

\section{LE CHEMINEMENT SCOLAIRE DES ÉTUDIANTS}

Les recherches sur l'évaluation de la réussite universitaire ont examiné l'influence de cinq groupes de variables : les caractéristiques sociodémographiques des étudiants, leur scolarité antérieure, leurs conditions de vie, leurs manières d'étudier et le contexte universitaire dans lequel ils étudient (Duru-Bellat, 1995 ; Romainville, 2000 ; Michaut, 2002). C'est à partir de ces cinq groupes, auxquels s'ajouteront les pratiques culturelles des étudiants, que l'analyse de la réussite s'effectuera (cf. schéma suivant). Pour évaluer la réussite, il apparaît indispensable d'aller audelà des simples tableaux de contingence en utilisant des analyses de régression multiples permettant de raisonner ceteris paribus, c'est-à-dire en tentant de s'affranchir des éventuels artefacts dus aux fortes corrélations entre certaines caractéristiques individuelles, et ce de manière à dégager les dimensions qui sont associées à la réussite (ou à l'échec) au cours des premières années d'études. La démarche repose uniquement sur l'estimation, à l'aide des régressions logistiques, de la probabilité de réussir la première année des beaux-arts (cf. modèle Logit en annexe) 
Figure 2. - Schéma de réussite dans les écoles supérieures d'art

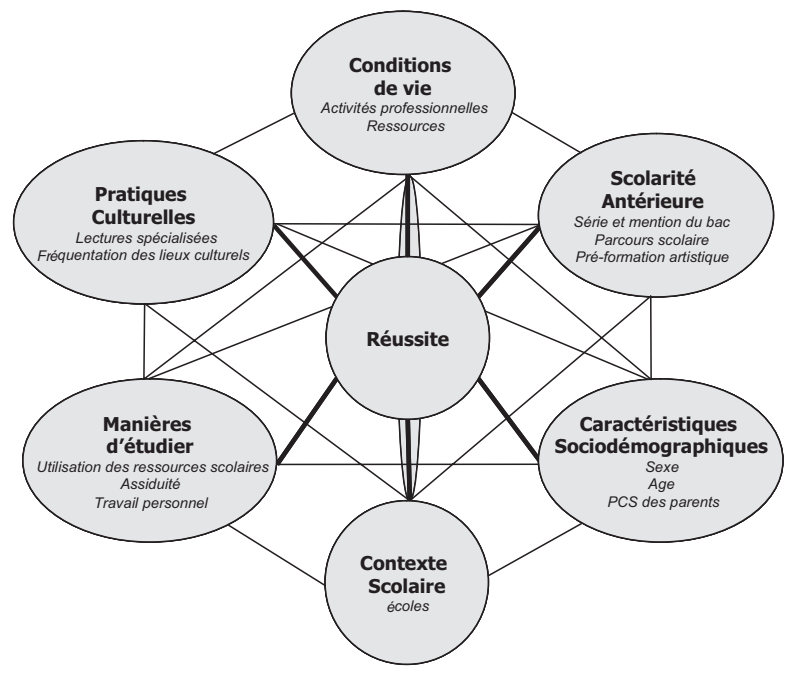

sachant que les écarts de réussite entre les différentes modalités de chaque variable sont plus prononcés en début de cursus qu'en deuxième année.

\section{La sélectivité des écoles d'arts}

Parmi les 1399 étudiants inscrits en $1^{\text {re }}$ année (4), près des trois quarts sont admis en $2^{\mathrm{e}}$ année, $6,4 \%$ redoublent et $21,3 \%$ échouent. Parmi ces derniers, plus d'un tiers abandonnent en cours d'année $(n=104)$ et $10 \%$ ne se présentent pas à l'intégralité des examens de fin d'année.

Ces résultats masquent la forte hétérogénéité de réussite selon les établissements. Ainsi, les taux d'obtention du Certificat d'études en arts plastiques (CEAP) en deux ans varient de $25 \%$ à Dunkerque à $87,1 \%$ à Orléans. Toutefois, ces différences de sélectivité omettent la sélectivité à l'entrée. Les écoles peuvent procéder à un recrutement très exigeant lors du concours d'accès et par la suite sélectionner très peu les étudiants. Ainsi, les écoles de Cergy, de Rennes et de Strasbourg sélectionnent peu les étudiants au cours des études mais sélectionnent fortement à l'entrée (environ un candidat sur six est retenu) (5). À l'inverse, l'école de Dunkerque accueille près des trois quarts des candidats mais sélectionne par la suite fortement les étudiants. Plus généralement, une tendance se dessine (matérialisée par la droite de régression linéaire figurant sur le graphique suivant) selon laquelle la sélectivité diminue lorsque les chances d'accès d'être d'admis au concours sont élevées.

\section{L'influence des caractéristiques sociodémographiques}

En raison des écarts de réussite importants entre les écoles, les modèles de régression tiennent compte du contexte d'étude. Parmi les caractéristiques sociodémographiques, seul le genre exerce un impact significatif pour l'admission en deuxième année alors qu'il n'existe aucune différence significative pour l'admission en troisième année. Plus précisément, on note que les filles réussissent davantage que les garçons. En ce qui concerne l'origine sociale, les enfants de cadre supérieur (y compris ceux qui exercent une profession de l'information, des arts et du spectacle) ne se distinguent pas des enfants des autres catégories sociales. La sélection sociale des étudiants semble donc s'opérer non pas au quotidien mais lors du recrutement des étudiants.

\section{L'influence des conditions de vie}

Dans quelle mesure les conditions de vie affectentelles le parcours des étudiants? On sait que les

Tableau II. - Taux de réussite des étudiants

\begin{tabular}{|l|r|c|c|}
\hline & Résultats en $1^{\text {re }}$ année & Résultats en 2de année & $\begin{array}{c}\text { Obtention du CEAP } \\
\text { en deux années }\end{array}$ \\
\hline Admis & $1011(72,3 \%)$ & $820(81,1 \%)$ & $820(58,6 \%)$ \\
\hline Redoublants & $90(6,4 \%)$ & & \\
\hline Insuccès (1) & $298(21,3 \%)$ & & $1399(100 \%)$ \\
\hline Total & $1399(100 \%)$ & $1011(100 \%)$ & $13 \%)$ \\
\hline
\end{tabular}

(1) Étudiants réorientés, échouant aux examens et non admis à redoubler, abandonnant, exclus ou ne se présentant pas aux examens. 
Figure 3. - La sélectivité des écoles supérieures d'art

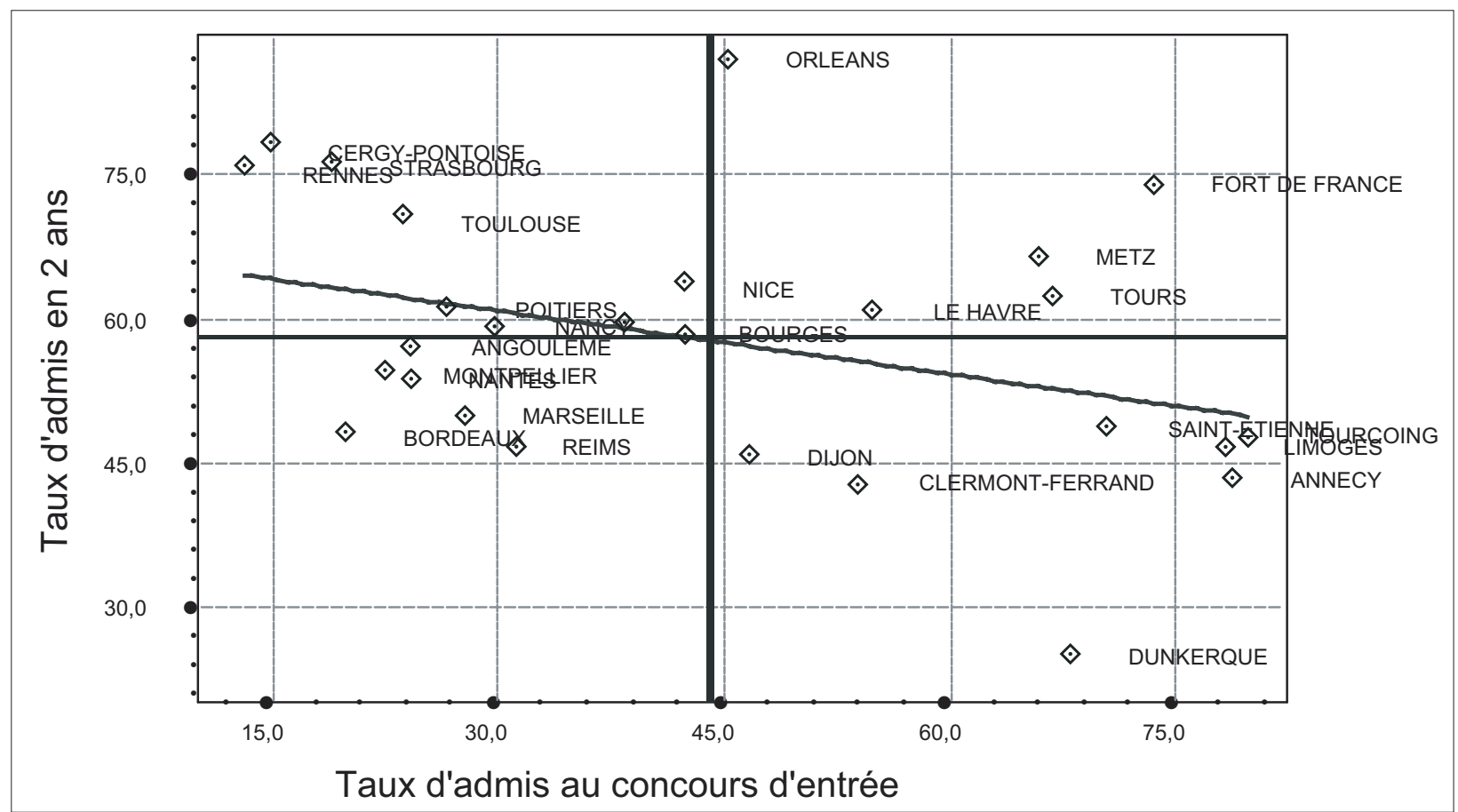

Lecture : L'école d'Amiens, qui représente la situation moyenne, retient $45 \%$ des candidats au concours d'entrée. $58 \%$ des étudiants de cette école obtiennent le CEAP $(B a c+2)$ en deux années.

ressources dont disposent les étudiants ont une incidence modeste sur leur cheminement (Grignon et Gruel, 1999 ; Gruel, 2002). Plus précisément, de faibles ressources parentales et une activité professionnelle régulière relativement intense (au moins à mi-temps) affectent les chances de réussite des étudiants. Cela étant, la scolarité antérieure et les manières d'étudier sont davantage associées à la réussite que les conditions de vie (Michaut, 2002), sachant toutefois que les étudiants de faible niveau scolaire sont plus susceptibles d'exercer une activité professionnelle de manière à réduire le coût d'opportunité des études. Ceci réduit d'autant leur chance d'obtenir leur diplôme. Les étudiants des beaux-arts n'échappent pas à cette situation. Certes, les étudiants déclarant avoir une activité professionnelle n'obtiennent pas de moins bons résultats en première année. Toutefois, plus cette activité s'intensifie, plus les chances de réussite diminuent. Les autres caractéristiques de la vie étudiante (ressources, type de logement) n'affectent pas la probabilité de réussir.

\section{Un passé scolaire faiblement associé à la réussite}

Alors que la scolarité antérieure est l'une des dimensions fondamentales de la réussite universitaire, elle n'exerce ici qu'un effet mineur. On constate effectivement que la réussite n'est pas significativement différente entre les différents bacheliers (série et mention). Les bacheliers technologiques ou professionnels ne sont pas défavorisés par rapport aux bacheliers généraux.

Ce résultat semble spécifique aux écoles supérieures d'art. Dans les universités, les taux de réussite en première année de DEUG artistique variaient en 1999 de $14,5 \%$ pour les bacs professionnels jusqu'à 53,9\% pour les bacs généraux en passant par $27,1 \%$ pour les bacs technologiques (Le Cosquer et Hérault, 2001).

Dans les écoles supérieures d'art, les étudiants qui n'ont pas obtenu de mention au bac ou qui ont redoublé au cours de leur scolarité n'apparaissent pas désavantagés. Seuls les redoublants obtiennent de meilleurs résultats par rapport aux bacheliers de l'an- 
née. Ceci étant, avoir reçu auparavant une formation artistique, que ce soit dans le cadre d'une école préparatoire ou dans une option au lycée, n'apporte pas une contribution significative à la réussite. En définitive, un préapprentissage de l'art n'est pas indispensable pour réussir des études supérieures d'art mais il est souvent nécessaire pour y accéder.

\section{La maîtrise du métier fait des différences}

Le fait que les redoublants réussissent mieux la première année révèle l'importance de l'intégration dans l'école. D'autres indicateurs corroborent ce résultat. Les étudiants les moins assidus, ceux qui ne travaillent jamais dans l'établissement réussissent significativement moins que les étudiants qui s'investissent fortement dans leurs études et les activités culturelles.

\section{De fortes variations de réussite selon les écoles}

Au total, le cheminement réussi des étudiants est davantage favorisé par l'intégration scolaire que par une scolarité antérieure d'excellence ou un héritage culturel. II dépend surtout du contexte scolaire. En effet, les écarts de réussite au Certificat d'études d'arts plastiques $(\mathrm{bac}+2)$ en deux ans varient selon les écoles de $25 \%$ à $87 \%$. Même lorsque sont contrôlées les caractéristiques personnelles et scolaires des étudiants, les écarts entre les écoles restent significatifs. Évidemment, ces résultats dépendent de la modalité de référence, à savoir, ici, une école présentant des taux de réussite dans la moyenne des écoles. Si l'école de référence est l'une des deux écoles dont les résultats sont les plus éloignés de la moyenne nationale (Dunkerque ou Orléans), alors les écarts sont pratiquement toujours significatifs. À noter que les écarts entre les écoles sont plus importants en $1^{\text {re }}$ année qu'en seconde année.

L'absence de relation significative entre le début du cheminement scolaire des étudiants et leur origine sociale ou leur héritage culturel va à contresens de l'approche "culturaliste » (Troger, 2002). Cela étant, les différences de composition sociale des écoles suggèrent comme source d'inégalité, des différences sociales d'orientation, en particulier dans le choix des écoles (6). Ce contexte scolaire apparaît très prégnant. D'une part, les conditions et les modalités de recrutement s'opèrent localement, aboutissant ainsi à une forte hétérogénéité sociale et scolaire du système. D'autre part, les différences de réussite entre les écoles et la faiblesse des déterminants "classiques" de la réussite dans l'enseignement supérieur (série et mention du bac par exemple) avalisent l'hypothèse d'un enseignement " contextualisé ". En atteste également l'absence d'effet des formations artistiques antérieurement reçues par les étudiants sur leur parcours ultérieur. Chaque école semble en définitive fonctionner selon ses propres critères de l'excellence artistique. La singularité comme source de valorisation individuelle se manifeste jusque dans les pratiques de sélection des écoles. Chacune d'entre elles distingue les étudiants selon des critères originaux excluant les formes traditionnelles de la méritocratie scolaire.

$$
\text { ** }
$$

Les écoles supérieures d'art ont connu, dans un sens, un mouvement inverse à celui des universités. Alors que ces dernières gagnent en autonomie (Musselin, 2001), les écoles supérieures d'art dont l'autonomie tient à leurs conditions mêmes d'existence, ont vu leur tutelle pédagogique se renforcer. Pour gagner en légitimité, elles durent s'aligner sur les conditions de reconnaissance des enseignements supérieurs, à savoir la construction de cursus nationaux et l'imposition d'un niveau d'étude minimum pour y accéder. Cela étant, l'hétérogénéité des écoles d'art en matière de statuts, de répartition territoriale ou de recrutement est telle que les politiques d'établissement semblent sinon s'imposer, du moins conserver une large place par rapport aux politiques tutélaires. Même s'il est vrai que ce secteur de l'enseignement supérieur n'est pas le seul concerné, les universités n'échappant pas totalement à cette hétérogénéité (Jarousse et Michaut, 2001). Dès lors, il n'est pas surprenant de constater des écarts importants de certification entre les écoles. II faut dire que la validation de la formation n'est pas exempte de toute ambiguïté. Si enseigner l'art constitue déjà un défi en soi, en matière de certification la spécificité de l'ambition devient redoutable. En effet, comment juger sans norme? Absence de référent, absence de corpus et enseignement non normé, chacun de ces éléments semble à lui seul en contradiction totale avec l'idée de diplôme. Les conceptions actuelles de l'art, primat de la singularité, expression souveraine de l'individu, production innovante et hors norme, sont autant d'éléments qui contrarient la recherche de critères de jugement et plus encore leur application.

Gilles Galodé IREDU-CNRS

Christophe Michaut CREN, Université de Nantes 
(1) Diplôme de fin d'étude du cycle long (5 ans) : DNSEP, diplôme supérieur national d'expression plastique. Diplôme de fin d'étude du cycle court (3 ans) : DNAT diplôme national d'art et techniques.

(2) L'ENSAD (Arts-déco. Paris) se distingue nettement des autres écoles en recrutant davantage les bacheliers situés au sommet de la hiérarchie scolaire, à savoir les bacheliers scientifiques ( $31,8 \%$ versus $11,7 \%$ en moyenne).
(3) À noter que L'ENSBA est membre de la conférence des grandes écoles.

(4) Les résultats des écoles parisiennes ne sont pas disponibles.

(5) À noter que les trois grandes écoles parisiennes (ENSCI, ENSBA, ENSAD) retiennent moins de $10 \%$ des candidats (source : Ministère de la culture, direction des enseignements artistiques).

(6) Pour s'en assurer, il faudrait comparer les caractéristiques des postulants et des candidats retenus.

\section{BIBLIOGRAPHIE}

AZAM M. (1998). - Parcours d'artistes ou le talent en questions. La reconnaissance et l'artiste en MidiPyrénées. Thèse de doctorat en Sociologie, Université Toulouse-Le-Mirail.

BOUDON R. (1973). - L'inégalité des chances. La mobilité sociale dans les sociétés industrielles. Paris : A. Colin.

COUSIN O. (2000). - Politiques et effets-établissements dans l'enseignement secondaire. In A. van Zanten (dir.), L'école : l'état des savoirs. Paris : La découverte.

DURU-BELLAT M. (1994). - Filles et garçons devant l'école. Revue française de pédagogie, $\mathrm{n}^{\circ} 109$, p. 111-143.

DURU-BELLAT M. (1995). - Des tentatives de prédictions aux écueils de la prévention en matière d'échec en $1^{\text {re }}$ année d'université. Savoir, $n^{\circ} 3$, p. 399-416.

DURU M., MINGAT A. (1988). - Les disparités de carrières individuelles à l'université : une dialectique de la sélection et de l'autosélection. L'Année sociologique, vol. 38, p. 309-339.

GALODÉ G. (1994). - Les écoles d'art en France. Évolution des structures d'offre et des effectifs. Dijon: Cahiers de l'Irédu, $\mathrm{n}^{\circ} 57$.

GALODÉ G. (2000). - Les élèves des écoles supérieures d'art. Les études dans les écoles supérieures d'art : déterminants de choix et cheminements de cohorte. Analyse longitudinale. Rapport d'étude pour le ministère de la Culture et de la Communication. Paris : DAP, $160 \mathrm{p}$.

GRIGNON C., GRUEL L. (1999). - La vie étudiante. Paris : PUF.

GRUEL L. (2002). - Les conditions de réussite dans l'enseignement supérieur. OVE infos, $n^{\circ} 2$, Observatoire de la Vie étudiante.
IMBERT J. (1998). - Une nouvelle place et un nouveau rayonnement pour les écoles d'art en France. Rapport de la Commission nationale d'étude sur les enseignements artistiques présidée par Jacques Imbert, présenté à Catherine Trautmann, ministre de la Culture et de la Communication. Paris : ministère de la Culture et de la Communication.

JAROUSSE J.-P., MICHAUT C. (2001). - Variété des modes d'organisation des premiers cycles et réussite universitaire. Revue française de pédagogie, $n^{\circ} 136$, p. 41-51.

LE COSQUER C., HÉRAULT D. (2001). - L'enseignement supérieur artistique et culturel. Année 1999-2000. Note d'information du ministère de l'éducation nationale, 01.14 .

LIOT F. (1999). - L'École des beaux-arts face aux politiques de soutien à la création. Sociologie du travail, $n^{\circ} 41$, p. 411-429.

MICHAUT C. (2002). - L'influence du contexte universitaire sur la réussite des étudiants. Lille : Presses universitaires du Septentrion.

MUSSELIN C. (2001). - La longue marche des universités françaises. Paris : PUF.

ROMAINVILLE M. (2000). - L'échec dans l'université de masse. Paris : L'Harmattan.

TROGER V. (2002). - La formation des artistes contemporains. Sciences Humaines, Hors-série n³7, p. 66-69.

SULZER É. (1999). - Apprendre l'Art. L'enseignement des arts plastiques et ses usages sociaux. Thèse de Doctorat en Sociologie, Université de Nantes. 
ANNEXES. - Modèle Logit. - La réussite en première d'année d'école supérieure d'art

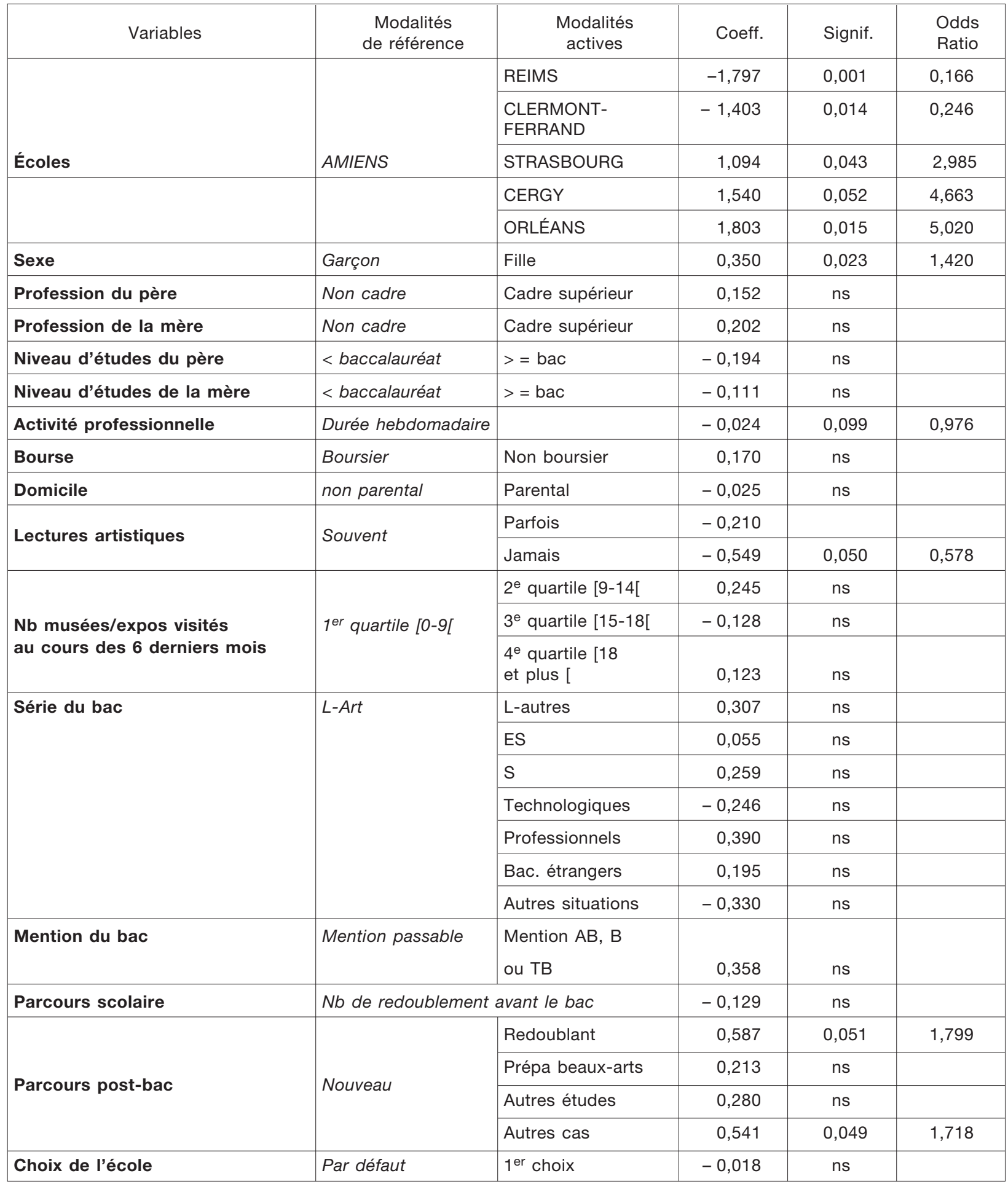




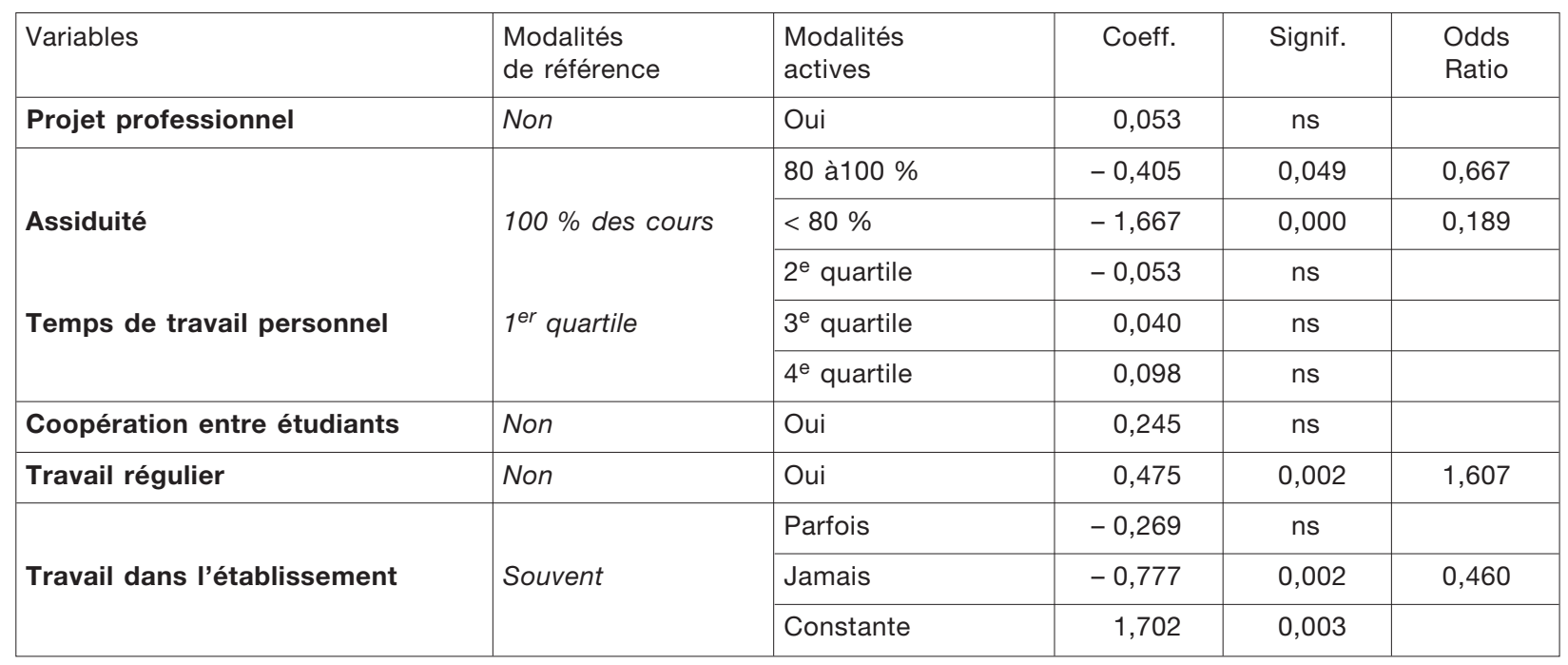

Lecture : l'odd ratio est égal à $\left(p_{a} / q_{a}\right) /\left(p_{b} / q_{b}\right)$ avec $p_{a} b=$ probabilité pour les groupes a et $\mathrm{b}$ d'atteindre un niveau et $q_{a} b=$ probabilité pour les groupes $a$ et $b$ de ne pas atteindre ce niveau. Par exemple, par rapport aux étudiants d'Amiens, les étudiants d'Orléans ont (67/3) / (40/9) $=5,02$ fois plus de chances d'être admis en deuxième année que d'être ajournés. Les odds ratio sont indiqués lorsque les écarts sont significatifs (seuil de probabilité du khi-carré inférieur à 0.10). 
